\title{
Performance of Savonius Rotor for Environmentally Friendly Hydraulic Turbine*
}

\author{
Miyoshi NAKAJIMA ${ }^{* *}$, Shouichiro IIO $^{* *}$ and Toshihiko IKEDA ${ }^{* *}$ \\ ${ }^{* \star}$ Department of Environmental Science and Technology, Shinshu University, \\ 4-17-1 Wakasato, Nagano 380-8553, Japan. \\ E-mail: shouiio@shinshu-u.ac.jp
}

\begin{abstract}
The aim of this investigation was to develop an environmentally friendly nano-hydraulic turbine. A model of a two-bucket Savonius type hydraulic turbine was constructed and tested in a water tunnel to arrive at an optimum installation condition. Effects of two installation parameters, namely a distance between a rotor and a bottom wall of the tunnel, a rotation direction of the rotor, on the power performance were studied. A flow field around the rotor was examined visually to clarify influences of installation conditions on the flow field. The flow visualization showed differences of flow pattern around the rotor by the change of these parameters. From this study it was found that the power performances of Savonius hydraulic turbine were changed with the distance between the rotor and the bottom wall of the tunnel and with a rotation direction of the rotor.
\end{abstract}

Key words: Environmentally Friendly Hydraulic Turbine, Savonius, Water Tunnel, Performance, Flow Visualization, Fluid Machinery

\section{Introduction}

Environmental issues as typified by global warming become conspicuous in recent years. It is obvious that achieving sustainable energy means using natural energy effectively. The hydropower should occupy the attention of electric power generating systems as it is clean and renewable energy sources with highest density, in cooperation with the wind and the solar powers. Most of hydropower is generated by a large-scale hydroelectric plant. Some have suggested that dam constructions can lead to the tremendous environmental damages. On the other hand, small/micro/nano hydropower has attracted much attention for recent years mainly because of decrease of construction place for large-scale plants and environmental conservation. The aim of this study is to develop a Savonius type hydraulic turbine to utilize effectively as a nano-hydropower and a dispersed power system. The turbine is suitable for rivers or irrigation canals in which the effective head is not sufficient while the flow rate is enough. It is thought that this approach could lead to cheaper power generation without the environmental disruptions, compared with that produced by the large-scale hydroelectric plant.

The Savonius rotor has been used mainly for a wind power ${ }^{(1)-(20)}$ and tidal/wave power generation ${ }^{(21)-(24)}$. There have been many studies of a Savonius wind rotor, e.g. rotor configurations ${ }^{(1)-(4)}$, a flow field around a rotor ${ }^{(5)-(15)}$, numerical simulations ${ }^{(15)-(20)}$ and others. The advantages of Savonius rotor using for the hydropower are little complex constitution, low cost, durability and easy maintenance. Although the previous studies give us useful information, problems of Savonius hydraulic turbine used for the nano-hydropower are still unclear. In the present study, the power performance is discussed and focused on the installation conditions: the distance between the rotor and the bottom 
wall, between the rotor and the water surface, the rotation direction of the rotor. In addition, we examined flow patterns around the rotor associated with power performances.

\section{Nomenclature}

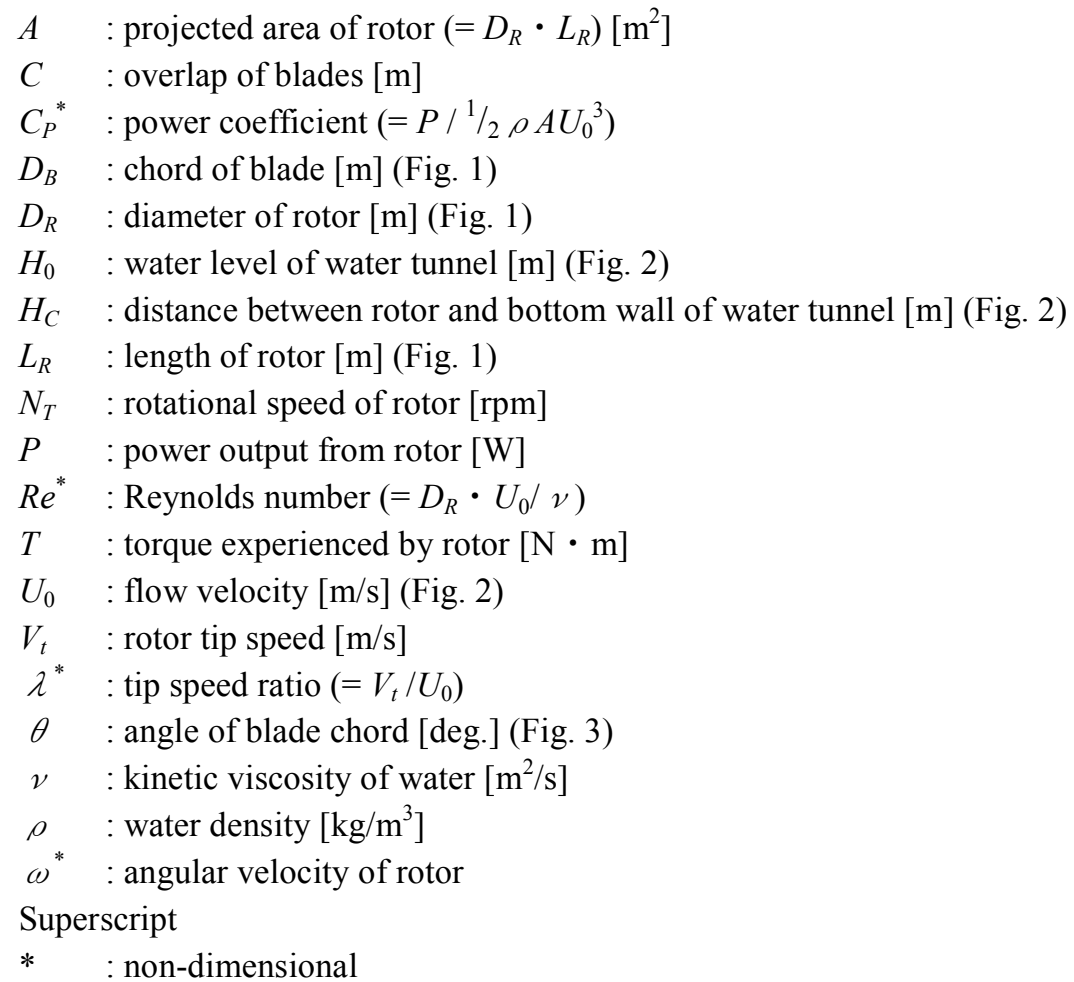

\section{Experimental apparatus and procedure}

A schematic of the test model orientation, instrumentation, load and measurement system is shown in Fig. 1. The rotor consists of rotating components (blades and endplates) held by bearings in the side plates and in the upper support structure. The rotor was constructed from stainless steel mounted on a $10.0 \mathrm{~mm}$ diameter steel rod. One end of the rotor shaft is coupled to a pulley and to upper mounted load (powder brake) and torque meter by a timing belt on pulleys. Torque performance is measured by strain gage type torque detector (KYOWA TP-10KCE) and strain amplifier (KYOWA HSC-20B). We began the measurement from the unloaded state at the supply voltage of $0 \mathrm{~V}$ to the powder brake and conducted the measurement by increasing the load until the rotor stopped. The blades are half cylinder with chord of $D_{B}=0.089 \mathrm{~m}$ and length of $L_{R}=0.21 \mathrm{~m}$. Overlap of the two blades is $C=0.032 \mathrm{~m}$, the dimensionless overlap ratio $C / D_{B}$ is 0.36 . The distance between the rotor and the side plate is $0.041 \mathrm{~m}$, and between the rotor and the upper plate is $0.046 \mathrm{~m}$. The performance of the rotor includes influence of the side plate and the upper plate in this experiment.

The tests were carried out in the circuit water tunnel. The test section is $0.6 \mathrm{~m}$ width, $0.5 \mathrm{~m}$ height and $3.0 \mathrm{~m}$ length with velocity variation in the empty tunnel up to $\pm 1.5 \%$. The section has a water surface like a field channel, the normal water level in the tunnel is set at $H_{0}=0.4 \mathrm{~m}$. The water-level difference between the rotor upstream and downstream side is $50 \mathrm{~mm}$. This drop induced the flow (water surface flow) illustrated later in Fig. 7. The projected area of the rotor is $0.03 \mathrm{~m}^{2}$, so that the rotor presented a tunnel blockage of $12 \%$. No tunnel interference corrections are included.

The detailed around the test section is shown in Fig. 2. The coordinate system is chosen in such a manner that the origin is located at cross point between a plumb line suspended 
from the center of the rotor and bottom of the tunnel, the $x$-axis is along the flow direction, the $y$-axis is in the horizontal direction perpendicular to the flow, and the $z$-axis is in the depthwise direction. The flow velocity at the center of the cross section of $x=-1000 \mathrm{~mm}$ is $U_{0}=0.8 \mathrm{~m} / \mathrm{s}$ (constant) which corresponds to a Reynolds numbers $\left(R e=U_{0} \cdot D_{R} / v\right)$ of $1.1 \times 10^{5}$, where $v$ is a kinematic viscosity of water. The flow velocity distribution at this cross section is uniform except for the region near the wall in a tunnel.
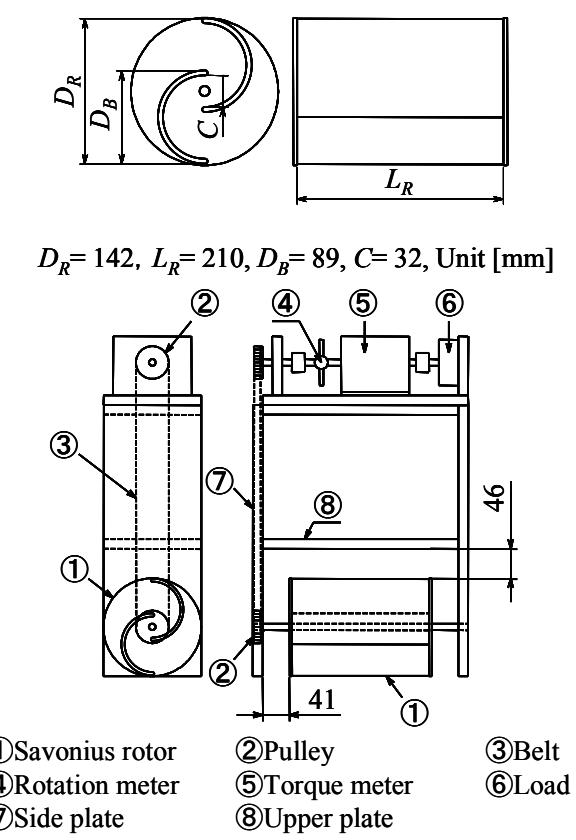

Fig. 1 Experimental apparatus and Savonius rotor

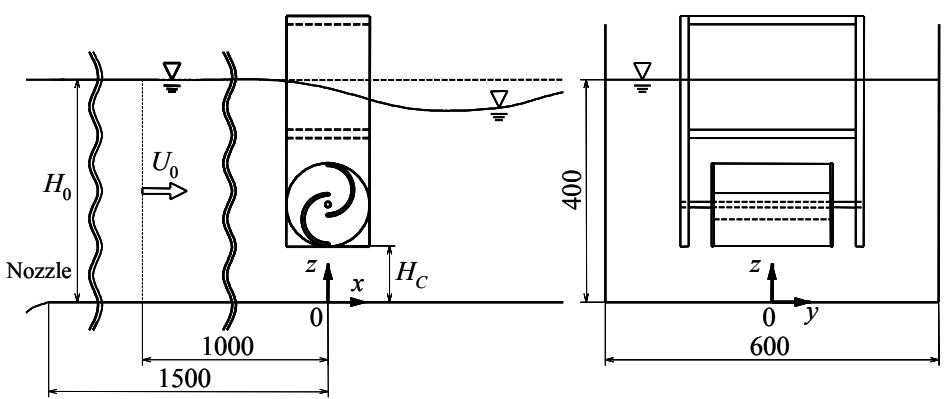

Fig. 2 Test section

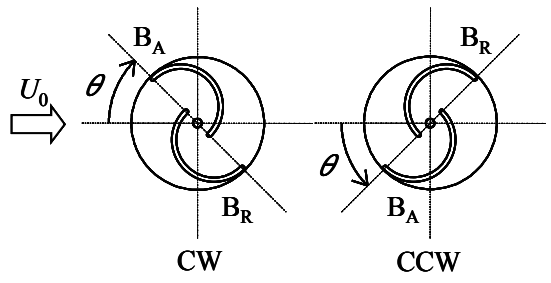

$\mathrm{B}_{\mathrm{A}}$ : Advancing blade, $\mathrm{B}_{\mathrm{R}}$ : Returning blade

Fig. 3 Rotation direction of rotor 
Figure 3 shows the definitions of a rotation direction of the rotor and the blade name. The clockwise rotation is defined as $\mathrm{CW}$, the counterclockwise direction is defined as CCW when main flow direction is from left to right. A rotation angle of the blade chord is defined as $\theta$ which corresponds to the main flow direction is $\theta=0^{\circ}$. A blade which located in the range $0^{\circ} \leq \theta<180^{\circ}$ is named of advancing blade, located in the range $180^{\circ} \leq \theta<360^{\circ}$ is named of returning blade. The distance between the rotor and the bottom wall of the tunnel is varied from 0.014 to $0.154 \mathrm{~m}$, and is defined as $H_{C}$.

\section{Experimental result}

\subsection{Measurement of the power coefficient}

The experiment was carried out in conditions shown as Table 1 . The $H_{C} / D_{R}$ is clearance ratio, and is changed from 0.10 to 1.08 . Figure 4 shows the effect of clearance ratio on the power performance in $\mathrm{CW}$ rotation. As can be seen from the graph, the curves exhibit a peak at $\lambda \sim 1.1$ in all clearance ratios. For $H_{C} / D_{R}=0.73$, the power coefficient is maximum. The power coefficient decreased with decreasing the clearance ratio as shown in this figure. This reason seems to be that the stagnation flow along the convex side of the returning blade increased the fluid drag due to some influence of the bottom wall of the water tunnel. The effect of clearance ratio on the power performance in CCW rotation is shown in Fig. 5. The curves exhibit a peak at $\lambda \sim 1.1$ in all clearance ratios except only for $H_{C} / D_{R}=0.10$. For $H_{C} / D_{R}=0.20$, the power coefficient is maximum. The power coefficient decreased with increasing the clearance ratio. This result is showed the opposite tendency in the case of $\mathrm{CW}$. This seems to be because the stagnation flow along the convex side of the returning

Table 1 Experimental conditions

\begin{tabular}{|c|c|}
\hline Rotation direction & $H_{C} / D_{R}$ \\
\cline { 1 - 2 } $\mathrm{CW}$ & $0.10,0.20,0.38,0.73,1.08$ \\
\hline $\mathrm{CCW}$ & \\
\hline
\end{tabular}

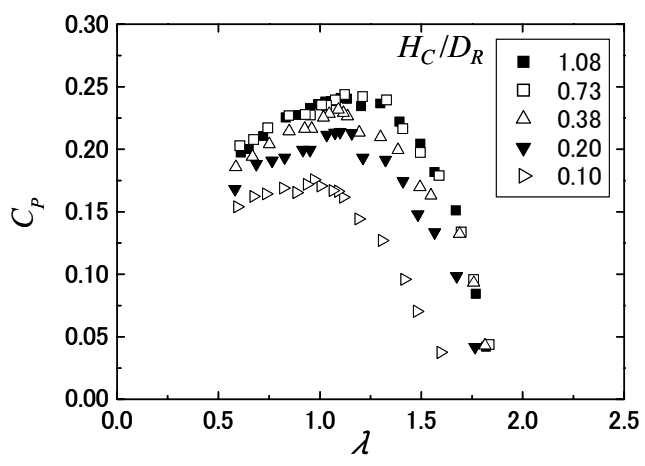

Fig. 4 Power performance for CW

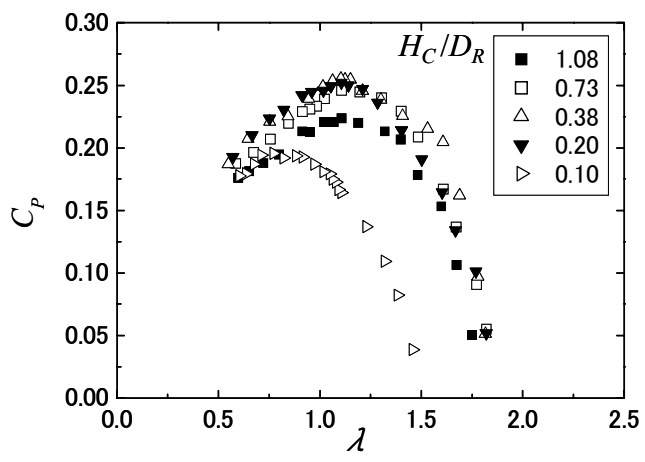

Fig. 5 Power performance for $\mathrm{CCW}$ 


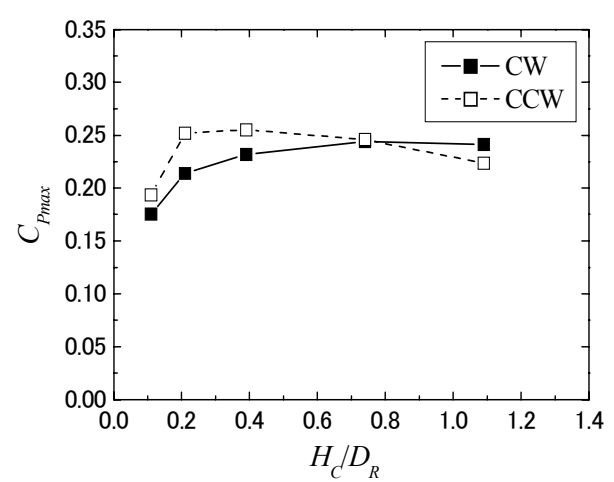

Fig. 6 Maximum power coefficient vs clearance ratio

blade increased the fluid drag as the rotor close to the water surface due to some influence of flow near the water surface. Ueno et al. studied the influence of a flat plate for wind collector on the performance of a Savonius wind rotor. They reported that the performance of the Savonius rotor improved with increasing the distance between the blade and the flat plate ${ }^{(20)}$. The flat plate is the equivalent of the bottom wall of the water tunnel in this study. It is the similar reason why the power performance changed with the distance between the rotor and the bottom wall.

The distribution of the maximum power coefficient, $C_{P \max }$, at the given clearance ratio is shown in Fig. 6. Now we compare $C_{P \max }$ for $\mathrm{CW}(\mathbf{\square})$ and the that for $\mathrm{CCW}$ ( $\square$ ). The value of $C_{P \max }$ is larger when the rotation direction is $\mathrm{CCW}$ for $H_{C} / D_{R}<0.73$. An important finding here is that the value of $C_{P \max }$ is larger when the advancing blade is near the water surface for $\mathrm{CW}$ and when the advancing blade is near the bottom wall for $\mathrm{CCW}$. The variation of $C_{P \max }$ with $H_{C} / D_{R}$ has a similar tendency in case of Savonius wind rotor ${ }^{(20)}$. From the measurement of the power coefficient, we could explain the increasing or decreasing tendency of $C_{P \max }$ with the change of $H_{C} / D_{R}$ and with the rotation direction, by considering the distance between the returning blade and the either of the bottom wall or the water surface.

\subsection{Visualization of flow around rotor}

To investigate the cause of the change in the power performances by the rotor-bottom distance or the rotation direction, we observed the flow field using the pigment streak-line method. For the pigment we used black ink used in fountain pens. We slowly injected the pigment into the flow field using a pipe with an external diameter $\phi 3.4 \mathrm{~mm}$ in order to avoid any influence on the rotor's characteristics. The injection point was set to $x=-200 \mathrm{~mm}$ on the central section of rotor width. A clear acrylic rotor of the same shape as the one used for the power performance measurement was employed for the visualization test. As the experimental conditions, the tip speed ratio was $\lambda \sim 1.1$ at which $C_{P \max }$ was obtained, the rotor was set at the height of $H_{C} / D_{R}=0.20$ or 1.08 , and the rotation direction of the rotor was $\mathrm{CW}$ or $\mathrm{CCW}$.

The results of the visualization $\left(\theta=0^{\circ}, 45^{\circ}, 90^{\circ}, 135^{\circ}\right)$ are shown in Fig. $7(\mathrm{a})$-(d), respectively. The ink was injected at the same height as the center of the rotation axis. In general the flows around a Savonius rotor are characterized as following patterns ${ }^{(10)-(19)}$. We therefore focused on these flow patterns.

( I ) Attached flow along the advancing blade's convex side

(II) Dragging flow from the advancing blade's convex side to the returning blade's concave side

(III) Through-flow in the overlapping area

(IV) Flow from the upstream of the rotor to the returning blade's convex side 
(V) Shedding vortex from the advancing blade's tip

(VI) Shedding vortex from the returning blade's tip

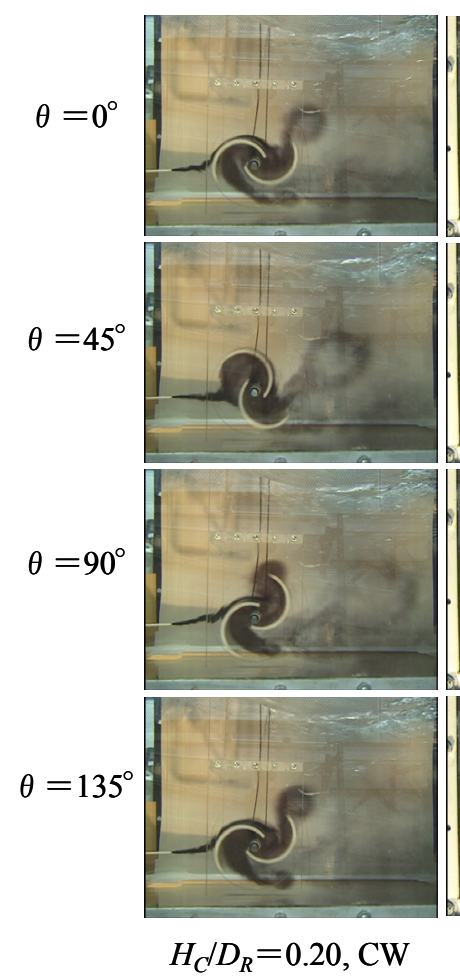

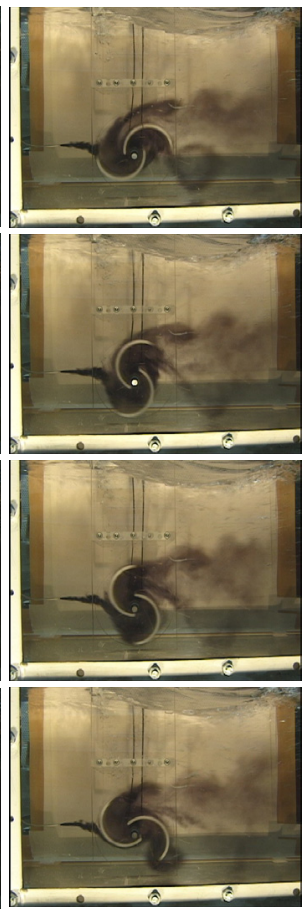

$H_{C} D_{R}=0.20, \mathrm{CCW}$
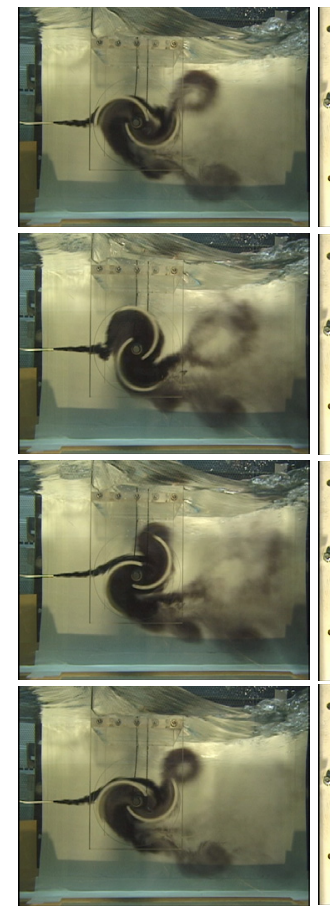

$H_{C} D_{R}=1.08, \mathrm{CW}$

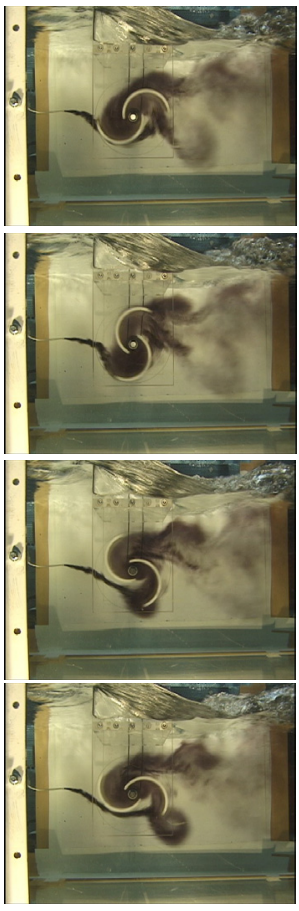

$H_{C} D_{R}=1.08, \mathrm{CCW}$

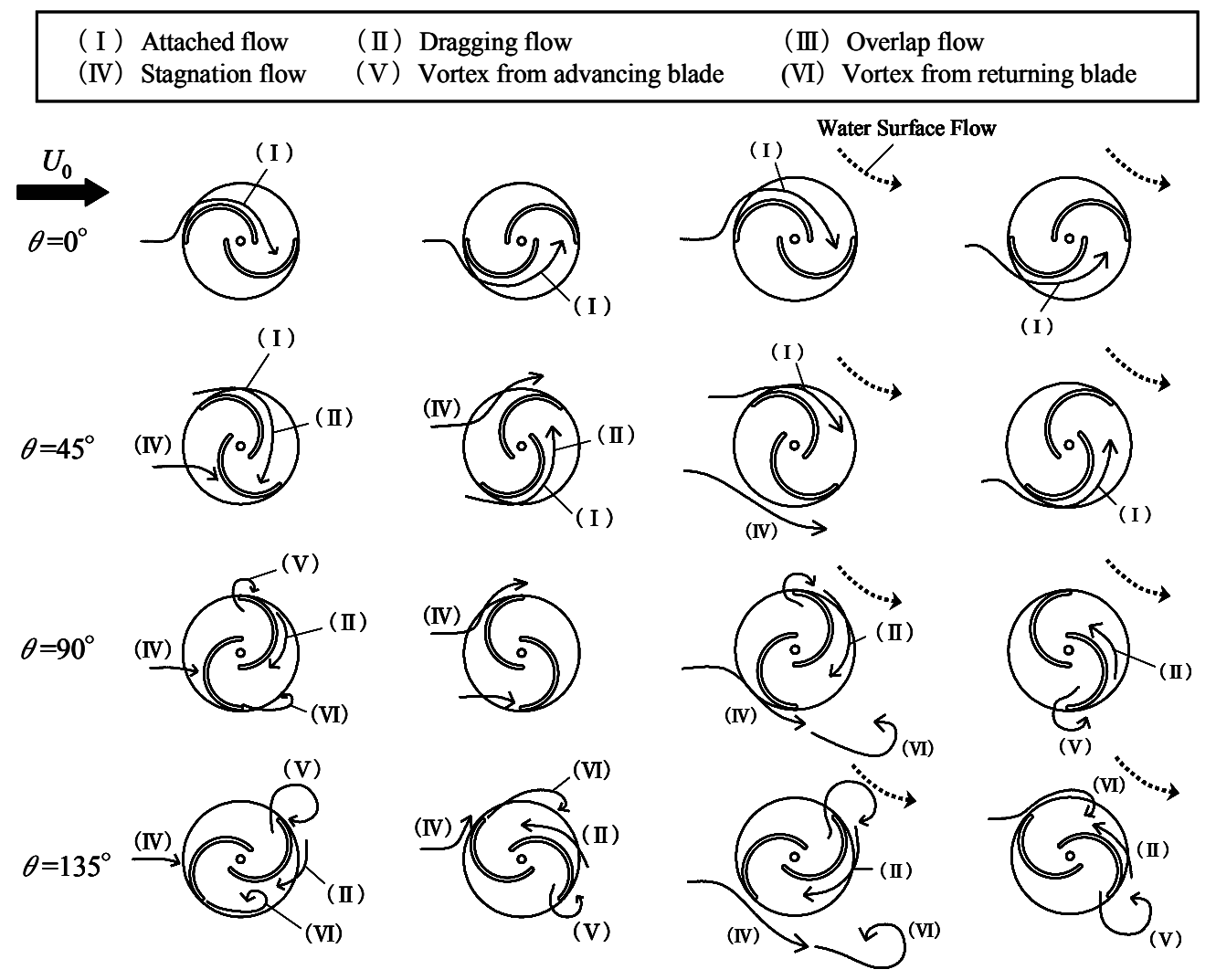

$\begin{array}{lll}\text { (a) } H_{C} / D_{R}=0.20, \mathrm{CW} & \text { (b) } H_{C} / D_{R}=0.20, \mathrm{CCW} & \text { (c) } H_{C} D_{R}=1.08, \mathrm{CW}\end{array}$

(d) $H_{C} / D_{R}=1.08, \mathrm{CCW}$

Fig. 7 Flow pattern around the rotor and flow model 
Flow ( I ) produces a lift, (II) and (III) restore the pressure on the returning blade's concave side, and (IV) affects the power reduction. The characteristic flow patterns around the rotor observed in the visualization are illustrated in the bottom of each figure. The symbols assigned to the arrows in the figures correspond to ( I ) to (VI) mentioned above.

Let us compare the flow patterns in Fig. 7(a) [CW] and 7(b) [CCW] for $H_{C} / D_{R}=0.20$ with which the rotor is located near the bottom wall of the tunnel. When $\theta \leq 45^{\circ}$, the attached flow ( I ) and the dragging flow ( II ) were observed for both rotation directions. When $\theta \geq 45^{\circ}$, there was a significant difference in flow (IV). In the case of CCW, this flow goes toward the upper part of the rotor to escape from the returning blade, while in the case of $\mathrm{CW}$ it stagnates in the upper part of the returning blade due to the small space between the rotor and the bottom wall and hence enhances the fluid drag against the returning blade. At $\theta=90^{\circ}$, the shedding vortex (V) is noticeable in Fig. 7(a) [CW]. In Fig. 7(b) [CCW], the flow field around the advancing blade is constrained by the bottom wall, and then the vortex generation ( V) at the blade's tip is suppressed. The vortex (VI) can be seen for both rotation directions and its generating position was closer to the advancing blade in Fig. 7(a) [CW] than in Fig. 7(b) [CCW], it could be due to the effect of the bottom wall of the tunnel. As a consequence, the dragging flow ( II ) to the concave side of the returning blade is blocked in Fig. 7(a) [CW], suppressing the pressure restoration effect. In the downstream side of the returning blade, the disturbance of the vortices is also observed. For the $\mathrm{CW}$ with $H_{C} / D_{R}=0.20$ where the rotor is located near the bottom wall, the flow field around the returning blade is constrained by the bottom wall and hence $C_{P}$ decreases with increasing of $\theta$.

We next compare the flow patterns in Fig. 7(c) [CW] and 7(d) [CCW] for $H_{C} / D_{R}=1.08$ where the rotor is located near the water surface. In this case, the water-level difference between the rotor upstream and downstream side is $50 \mathrm{~mm}$. This drop induced the flow (water surface flow) illustrated by an arrow in Fig. 7. The attached flow (I) and the dragging flow ( II ) are observed for both rotation directions. For CW, as the rotor's rotation direction coincides with the direction of the water surface flow induced by the water-level difference, flows ( I ) and (II) are both enhanced. Therefore, $C_{P}$ increases with increase of the lift and of the pressure restoration effect. For CCW, as the rotor's rotation direction is opposite to the water surface flow direction, the fluid drag against the returning blade increases and $C_{P}$ decreases. There is also a significant difference in the vortex (VI) at $\theta>45^{\circ}$ between both rotation directions. The vortex (VI) is clearly observed for $\mathrm{CW}$ but its generating position is below the returning blade and hence does not interfere with the dragging flow (II ). For CCW, however, the vortex is generated near the convex side of the advancing blade due to the water surface flow, and hence blocks the dragging flow ( II ).

Next we compare the results of Fig. 7(a) $\left[H_{C} / D_{R}=0.20\right]$ and Fig. 7 (c) $\left[H_{C} / D_{R}=1.08\right]$. When $H_{C} / D_{R}$ is large (Fig. 7(c)), there is a sufficiently large space between the rotor and the bottom wall, and even when $\theta$ increases flow (IV) would not stagnate and goes into the lower area of the rotor through the clearance between the rotor and the bottom wall. The vortex (VI) generated when $\theta \sim 90^{\circ}$, develops in the downstream side of the lower area as $\theta$ increases. The vortex (VI) strengthens the dragging flow (II ) when $\theta \geq 90^{\circ}$, enhancing the pressure restoration effect. On the other hand the advancing blade moves closer to the water surface. For CW, as the rotor's rotation direction coincides with the direction of the water surface flow, the attached flow ( I ) is strengthened when $\theta<90^{\circ}$ and the dragging flow ( II ) is strengthened when $\theta \geq 90^{\circ}$. For $\mathrm{CW}$, with the increasing $H_{C} / D_{R}$, the effect of the bottom wall on the returning blade is decreasing. In contrast, the effect of the water surface flow on the advancing blade is increasing. Thus the fluid drag against the returning blade becomes weaker and the attached flow on the advancing blade is strengthened, $C_{P}$ for $\mathrm{CW}$ could increase with increasing $H_{C} / D_{R}$. 


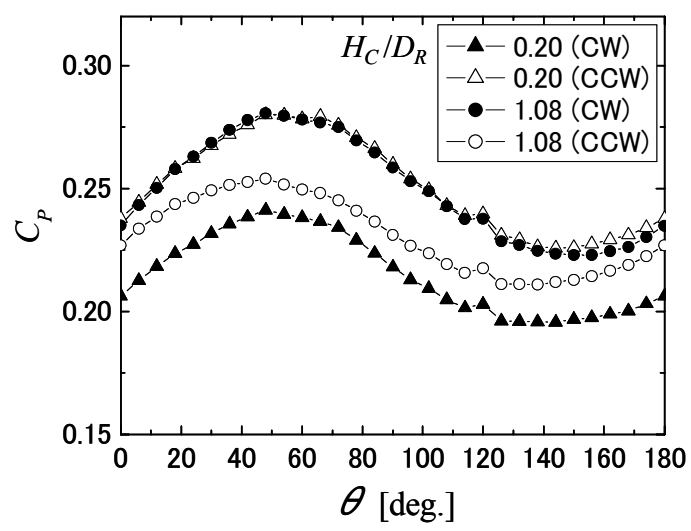

Fig. 8 Dynamic performance

\subsection{Dynamics evaluation of power performance}

In the previous section we mentioned that rotor-bottom distance and rotation direction of the rotor affect the power performances. Here we evaluate the effect for different rotation angles of the blade chord. We measured the torque and rotation frequency by changing $\theta$ by $6^{\circ}$ at $\lambda \sim 1.1$ that gave the highest $C_{P}$, and then calculated $C_{P}$ for $0^{\circ} \leq \theta \leq 180^{\circ}$. The measurements were conducted for three minutes for each condition, and an ensemble average was derived from the data of approximately 720 cycles.

For $\mathrm{CW}$ and $\mathrm{CCW}$, the power coefficients with $H_{C} / D_{R}=0.20$ and 1.08 are shown in Fig. 8. The change of $C_{P}$ by the change of $\theta$ has a similar tendency in any experimental conditions. The value of $C_{P}$ increases when $\theta<45^{\circ}$ and reaches the maximum when $\theta \sim 45^{\circ}$. It then decreases with $\theta$ larger than $45^{\circ}$ and reaches the minimum when $\theta \sim 135^{\circ}$. The increase of $C_{P}$ could be caused by the lift that is generated by the attached flow for $0^{\circ}<\theta<45^{\circ}$ as shown in the visualization in the previous section. When $90^{\circ}<\theta<135^{\circ}$, the vortex generation at the advancing blade's tip causes the separation of the attached flow on the advancing blade's convex side and hence $C_{P}$ is decreased because of reducing the lift. $C_{P}$ reaches the minimum value at $\theta \sim 135^{\circ}$, since the stagnation point moves outward on the returning blade's surface, and then the fluid drag against the returning blade is increased as reported by Fujisawa et al ${ }^{(11)}$.

Now we compare $C_{P}$ for $\mathrm{CW}(\mathbf{\Delta})$ and that for $\mathrm{CCW}(\triangle)$ with $H_{C} / D_{R}=0.20 . C_{P}$ is always larger when the rotation direction is $\mathrm{CCW}$, irrespective of $\theta$. For $\mathrm{CCW}$, the flow at the clearance between the rotor and the bottom wall is accelerated. This leads to the increasing of the fluid drag on the advancing blade's concave side for $0^{\circ}<\theta<180^{\circ}$. Since the accelerated flow suppresses the vortex generation from the advancing blade's tip, the separation of the attached flow on the advancing blade's convex side is prevented for $135^{\circ}<\theta<180^{\circ}$. Additionally, the stagnation flow flows to the rotor upper area along the returning blade's convex side, and then the fluid drag does not almost act on this convex side for $45^{\circ}<\theta<135^{\circ}$. For $\mathrm{CW}$, the flow through the clearance under the rotor increases the fluid drag on the returning blade's convex side for $0^{\circ}<\theta<180^{\circ}$. The vortex from the returning blade's tip blocks the dragging flow along the advancing blade's convex side, so the effect of pressure restoration at the returning blade's concave side for $135^{\circ}<\theta<180^{\circ}$. These could explain why the $C_{P}$ is always larger for $\mathrm{CCW}$ than for $\mathrm{CW}$.

When $H_{C} / D_{R}=1.08$, on the other hand, $C_{P}$ is always larger for $\mathrm{CW}(\mathbf{)})$ than for $\mathrm{CCW}$ (○). For CW, the water surface flow illustrated in Fig. 7 strengthens the attached flow on the advancing blade's convex side, and then the lift increases for $0^{\circ}<\theta<45^{\circ}$. The flow to the advancing blade's concave side also is strengthened by the water surface flow, so the torque toward clockwise direction increases for $90^{\circ}<\theta<180^{\circ}$. Additionally, the stagnation flow flows to the rotor lower area along the returning blade's convex side, and then the fluid drag does not almost act on this convex side for $45^{\circ}<\theta<135^{\circ}$. For CCW, the fluid drag on the returning blade's convex side is enhanced by the water surface flow for $0^{\circ}<\theta<90^{\circ}$. The vortex from the returning blade's tip blocks the dragging flow along the advancing blade's convex side, so the effect of pressure restoration at the returning blade's concave side 
decreases for $135^{\circ}<\theta<180^{\circ}$.

From the evaluation of dynamic performance, we could explain the increasing or decreasing of $C_{P}$ with the change of $\theta$, by considering the flow field around the rotor. We found that the change of the flow field around the rotor caused by the bottom wall of the tunnel and the water surface was the direct cause for the change in the performances by the rotor-bottom distance or the rotation direction of the rotor.

\section{Conclusions}

This study has explored the effect of the two installation parameters, namely distance between the rotor and bottom wall of the tunnel, rotation direction of the rotor, on the power performances. The flow field around the rotor was examined visually to clarify influences of installation conditions on the flow field. The following conclusions can be drawn:

(1) The power coefficient of Savonius rotor used for nano-hydraulic turbine is affected by varying the clearance ratio, $H_{C} / D_{R}$. The maximum power coefficient, $C_{P \max }$, is larger when the rotation direction is $\mathrm{CCW}$ for $H_{C} / D_{R}<0.73$, while $C_{P \max }$ is larger when the rotation direction is $\mathrm{CW}$ for $H_{C} / D_{R}>0.73$.

(2) The flow passing the rotor and the bottom wall of the tunnel is important factor for power performances when the rotor set in lower position. For $\mathrm{CW}$, the stagnation flow on the convex side of the returning blade enhances the fluid drag against the returning blade. The shedding vortex generated near the tip of the returning blade blocked the dragging flow to the concave side of the returning blade, and suppressed the pressure restoration effect.

(3) The flow near the water surface is important factor for power performances when the rotor set in higher position. For $\mathrm{CW}$, the flow enhances the attached flow on the convex side of the advancing blade, and strengthens the lift against the advancing blade. For CCW, on the other hand, the returning blade goes to the flow, and gives rise to enhance the fluid drag against the returning blade.

\section{Acknowledgement}

This work was supported partly by the Grant-in-Aid for Exploratory Research under Grant No.19651033 from Japan Society for the Promotion of Science.

\section{References}

(1) Takagi, K. and Endo, Y., Experimental study of a Savonius Windmill, Turbomachinery, Vol.4, No.2 (1976), pp.136-139 (in Japanese).

(2) Sheldahl, R. E., Feltz, L. V. and Blackwell, B. F., Wind Tunnel Performance Data for Two and Three-Bucket Savonius Rotors, Journal of Energy, Vol.2, No.3 (1978), pp.160-164.

(3) Sivasegaram, S., Secondary Parameters Affecting the Performance of Resistance-type Vertical-axis Wind Rotors, Wind Engineering, Vol.2, No.1 (1978), pp.49-59.

(4) Ushiyama, I., Nagai, H. and Shinoda, J., Experimentally Determining the Optimum Design Configuration for Savonius Rotors, Transactions of the Japan Society of Mechanical Engineers, Series B, Vol.52, No.480 (1986), pp.2973-2981.

(5) Bowden, G. J. and McAleese, S. A., The Properties of Isolated and Coupled Savonius Rotors, Wind Engineering, Vol.8, No.4 (1984), pp.271-288.

(6) Bergeles, G. and Athanassiadis, N., On the Flow Field around a Savonius Rotor, Wind Engineering, Vol.6, No.3 (1982), pp.140-148.

(7) Murai, H., Kataoka, M., Narasaka, T., Watanabe, H. and Onuma, S., Influence of Flaps and Deflectors on Power and Torque Characteristics of Savonius Rotor, Journal of the Visualization Society of Japan, 4-Suppl. (1984), pp.11-16 (in Japanese).

(8) Sawada, T., Nakamura, M. and Kamada, S., Blade Force Measurement and Flow Visualization of Savonius Rotors, Transactions of the Japan Society of Mechanical Engineers, Series B, Vol.51 No.471 (1985), pp.3743-3747. 
(9) Fujisawa, N. and Shirai, H., Measurement of Fluctuation Velocity around a Savonius Rotor in Open Type Wind Tunnel, Turbomachinery, Vol.14, No.6 (1985), pp.321-326 (in Japanese).

(10) Fujisawa, N., Shirai, H. and Mizuno, Y., Measurement of Unsteady Flow Pattern around a Savonius Rotor, Turbomachinery, Vol.14, No.11 (1986), pp.658-663 (in Japanese).

(11) Fujisawa, N., Shirai, H. and Saikawa, Y, Visualization of Unsteady Flow-Pattern around a Savonius Rotor, Flow Visualization, Vol.7, No.25 (1987), pp.107-111 (in Japanese).

(12) Fujisawa, N., Shirai, H. and Saikawa, Y., Investigation of the Field and Power Mechanism of a Savonius Rotor: Flow Visualization by a Smoke-wore Method, Transactions of the Japan Society of Mechanical Engineers, Series B, Vol.53, No.496 (1987), pp.3716-3721.

(13) Fujisawa, N., Experimental Investigation of the Unsteady Flow Field around a Savonius Rotor at Maximum Power Performance, Wind Engineering, Vol.11, No.4 (1987), pp.195-206.

(14) Fujisawa, N. , Ogawa, Y. and Shirai, H., Flow Field inside a Savonius Rotor and Its Power Mechanism, Transactions of the Japan Society of Mechanical Engineers, Series B, Vol.56, No.531 (1990), pp.3403-3407.

(15) Fujisawa, N. and Taguchi, Y., Investigation of Flow Field around Savonius Rotors by Particle Imaging Velocimetry and Numerical Calculations, Turbomachinery, Vol.23, No.8 (1995), pp.439-447.

(16) Ogawa, T., Research of a Savonius Rotor: Part 1 Theoretical Analysis, Transactions of the Japan Society of Mechanical Engineers, Series B, Vol.49, No.441 (1983), pp.976-984.

(17) Ishimatsu, K., Shinohara, T. and Takuma, F., Numerical Simulation for Savonius Rotors: Running Performance and Flow Fields., Transactions of the Japan Society of Mechanical Engineers, Series B, Vol.60, No.569 (1994), pp.154-160.

(18) Ishimatsu, K., Shinohara, T., Kage, K. and Okubayashi, T., Numerical Simulation for Savonius Rotors (Effects of Shed Vortices on Running Performance), Transactions of the Japan Society of Mechanical Engineers, Series B, Vol.61, No.581 (1995), pp.12-17.

(19) Yamagishi, M., Phase Averaging Analysis of Velocity Profiles in the Wake of a Savonius Rotor, Journal of the Visualization Society of Japan, Vol.24, Suppl. No.1 (2004), pp.79-82.

(20) Ueno, H., Mino, M. and Takada, N., Savonius type wind turbine: Influence of a side plate, Journal of Japan Solar Energy Society, Vol.29, No.4 (2002), pp.35-40.

(21) Higo, T., Takasugi, Y., Fujiwara, T. and Noguchi, H., Efficiency of Savonius Hydro Turbine for Tidal Current (in Japanese), Reports of the Government Industrial Research Institute Chugoku, No.32 (1989), pp.1-13.

(22) Tan, S., Shimizu, Y. and Kikuyama, K., Experimental Studied on a Savonius Rotor with Casing, Transactions of the Japan Society of Mechanical Engineers, Series B, Vol.63, No.611 (1997), pp.2356-2363.

(23) Katsuhara, K., Kitamura, F., Kajiwara, K. and Ohta, Y., Characteristics of Air Turbine for Light Beacon Generating from Wave Power: Impulse, Wells and Savonius Type (in Japanese), Japan Marine Science and Technology Center, (1987), pp.83-91.

(24) Okuda, K., Watabe, T., Kondoh, H. and Yano, K., Study of the Wave Energy Conversion System Fixed on the Seashore: Part 1 A Water Turbine of Savonius Type for Wave Power, Memoirs of the Muroran Insutituteof Technology, (1981), pp.427-432. 